\title{
What can we learn from GRBs?
}

\author{
Marco Muccino ${ }^{1,2, \star}$, Remo Ruffini ${ }^{1,2,3,4}$, Yerlan Aimuratov ${ }^{1,3}$, Laura M. Becerra ${ }^{1,2}$, Carlo L. Bianco ${ }^{1,2}$, Mile Karlica $^{1,3}$, \\ Milos Kovacevic ${ }^{1,3}$, Julio D. Melon Fuksman ${ }^{1,2}$, Rahim Moradi ${ }^{1,2}$, Ana V. Penacchioni ${ }^{5,6}$, Giovanni B. Pisani ${ }^{1,2}$, \\ Daria Primorac ${ }^{1,2}$, Jorge A. Rueda ${ }^{1,2,4}$, Soroush Shakeri ${ }^{2,7}$, Gregory V. Vereshchagin ${ }^{1,2}$, She-Sheng Xue ${ }^{1,2}$, and \\ Yu Wang ${ }^{1,2}$ \\ ${ }^{1}$ Dip. di Fisica, Sapienza Università di Roma, Piazzale Aldo Moro 5, I-00185 Rome, Italy \\ ${ }^{2}$ ICRANet-Pescara, Piazza della Repubblica 10, I-65122 Pescara, Italy \\ ${ }^{3}$ Université de Nice Sophia-Antipolis, Grand Château Parc Valrose, Nice, CEDEX 2, France \\ ${ }^{4}$ ICRANet-Rio, Centro Brasileiro de Pesquisas Fisicas, Rua Dr. Xavier Sigaud 150, Rio de Janeiro, RJ, 22290-180, Brazil \\ ${ }^{5}$ University of Siena, Dept. of Physical Sciences, Earth and Environment, Via Roma 56, I-53100 Siena, Italy \\ ${ }^{6}$ ASI Science Data Center, via del Politecnico s.n.c., I-00133 Rome Italy \\ ${ }^{7}$ Department of Physics, Isfahan University of Technology, 84156-83111, Iran
}

\begin{abstract}
We review our recent results on the classification of long and short gamma-ray bursts (GRBs) in different subclasses. We provide observational evidences for the binary nature of GRB progenitors. For long bursts the induced gravitational collapse (IGC) paradigm proposes as progenitor a tight binary system composed of a carbon-oxygen core $\left(\mathrm{CO}_{\text {core }}\right)$ and a neutron star (NS) companion; the supernova ( $\mathrm{SN}$ ) explosion of the $\mathrm{CO}_{\text {core }}$ triggers a hypercritical accretion process onto the companion NS. For short bursts a NS-NS merger is traditionally adopted as the progenitor. We also indicate additional sub-classes originating from different progenitors: $\left(\mathrm{CO}_{\text {core }}\right)$-black hole $(\mathrm{BH}), \mathrm{BH}-\mathrm{NS}$, and white dwarf-NS binaries. We also show how the outcomes of the further evolution of some of these sub-classes may become the progenitor systems of other sub-classes.
\end{abstract}

\section{Introduction}

Gamma-ray bursts (GRBs) are traditionally classified based on their duration [1-5]: short GRBs last $\lesssim 2$ s, while long GRBs last $\gtrsim 2 \mathrm{~s}$.

Thanks to the extensive data collected by $\gamma$-ray telescopes, such as AGILE, BATSE, BeppoSAX, Fermi, HETE-II, INTEGRAL, Konus/WIND and Swift, to more sofisticated time-resolved spectral analyses, and to the theoretical treatment of the fireshell model [6-8] it has become evident that both long and short bursts originate from binary progenitors and that they can be further subdivided into a variety of sub-classes, depending on the evolution of these binary systems [9-11].

Short bursts are associated to NS-NS or BH-NS mergers [12-22]: their host galaxies are of both early- and latetype, their localization with respect to the host galaxy often indicates a large offset [23-29] or a location of minimal star-forming activity with typical circumburst medium (CBM) densities of $\sim 10^{-5}-10^{-4} \mathrm{~cm}^{-3}$, and no supernovae ( $\mathrm{SNe}$ ) have ever been associated to them.

Long bursts have been traditionally associated to the death of single massive stars [30]. The large majority of long bursts is related to $\mathrm{SNe}$ and are spatially correlated with bright star-forming regions in their host galaxies $[31,32]$ with a typical CBM density of $\sim 1 \mathrm{~cm}^{-3}[33,34]$. However, the above single progenitor model contrasts with

\footnotetext{
^e-mail: marco.muccino@icranet.it
}

the fact that most massive stars are found in binary systems [35], that most type Ib/c SNe occur in binary systems [36] and that $\mathrm{SNe}$ associated to long GRBs are indeed of type $\mathrm{Ib} / \mathrm{c}$ [37]. Indeed, recently we have found evindence for multiple components in long GRB emissions evidencing the presence of a precise sequence of different astrophysical processes [33, 34], which led to the formulation of the Induced Gravitational Collapse (IGC) paradigm [6, 3840] expliciting the role of binary systems as progenitors of the long GRBs. The IGC paradigm explains the GRB-SN connection by proposing as progenitors (or in-state) a tight binary system composed of a carbon-oxygen core $\left(\mathrm{CO}_{\text {core }}\right)$ undergoing a SN explosion and a companion neutron star (NS) [39-41]. The SN explosion triggers hypercritical accretion onto the companion NS [39, 40, 42].

Recent observations of a prolonged $0.1-100 \mathrm{GeV}$ high energy emission by the Fermi-LAT instrument evidenced its correlation with both long [9] and short bursts [10] with isotropic energy $E_{\text {iso }} \gtrsim 10^{52} \mathrm{erg}$. On the basis of this correlation in such systems with different progenitors, we have identified the onset of the $\mathrm{GeV}$ emission with the moment of the formation of a black hole (BH) $[9,10]$. This implies that systems with energy $E_{\text {iso }} \lesssim 10^{52}$ erg, which do not exhibit $\mathrm{GeV}$ emission, do not form BHs.

Indeed, we proposed the following classification scheme. Long GRBs, according to the IGC paradigm [9], are classified into two sub-classes [42]: 
- X-ray flashes (XRFs) with $E_{\text {iso }} \lesssim 10^{52} \mathrm{erg}$ and restframe spectral peak energy $E_{p, i} \lesssim 200 \mathrm{keV}$. These systems, already pioneered in a different context [43-45], originate in widely separated $\mathrm{CO}_{\text {core }}-\mathrm{NS}$ binaries with an orbital separation $a>10^{11} \mathrm{~cm}$ [41], therefore the hypercritical accretion onto the NS companion is insufficient to induce gravitational collapse to a $\mathrm{BH}$ [39-41] and, therefore, as expected in our theory no GeV emission is observed. The outcomes (or out-states) of XRFs are binaries composed of a new NS $(v \mathrm{NS})$ produced from the SN explosion, and a massive NS (MNS) which accreted matter from the SN ejecta. Their occurrence rate is $\rho_{\mathrm{XRF}}=100_{-34}^{+45} \mathrm{Gpc}^{-3} \mathrm{yr}^{-1}$ [42] (see figure 1).

- Binary-driven hypernovae (BdHNe) with $E_{\text {iso }} \gtrsim$ $10^{52} \mathrm{erg}$ and $E_{p, i} \gtrsim 200 \mathrm{keV}$. BdHNe occur in tighter binaries $\left(a<10^{11} \mathrm{~cm}\right)$, where the hypercritical accretion onto the companion NS leads to the formation of a BH [41] and, therefore, to the emission of the associated prolonged $\mathrm{GeV}$ emission (observable when inside the LAT field of view). Specific constant power-law behaviors are observed in their high energy GeV and Xrays luminosity light curves $[9,46,47]$. The out-states of BdHNe are $v \mathrm{NS}-\mathrm{BH}$ binaries [41, 42, 48, 49]. The BdHN occurrence rate is $\rho_{\mathrm{BdHN}}=0.77_{-0.08}^{+0.09} \mathrm{Gpc}^{-3} \mathrm{yr}^{-1}$ [42] (see figure 1).

For progenitor system composed of a $\mathrm{CO}_{\text {core }}$ in binary with an already formed $\mathrm{BH}[8,42]$, leading to bursts with $E_{\text {iso }} \gtrsim 10^{54} \mathrm{erg}$ and $E_{p, i} \gtrsim 2 \mathrm{MeV}$, the observational identification is still pending. In these systems, which correspond to the late evolutionary stages of X-ray binaries as Cyg X-1 and Cyg X-3 [50], or microquasars [51], the hypercritical accretion produces, as out-states, a more massive $\mathrm{BH}$ and a $v \mathrm{NS}$. Their occurrence rate can contribute to that of $\mathrm{BdHNe}$, being $\mathrm{CO}_{\text {core }}-\mathrm{BH}$ a particular case of BdHN progenitors (see figure 1).

In total analogy, the formation of a $\mathrm{BH}$ can occur in short bursts, depending whether or not the mass of the merged core of the binary system exceeds the NS critical mass. For NS-NS binaries, which are the outcomes of XRFs, we have [10, 42, 48]:

- Short gamma-ray flashes (S-GRFs), with $E_{\text {iso }} \lesssim 10^{52} \mathrm{erg}$ and $E_{p, i} \lesssim 2 \mathrm{MeV}$. They occur when the merged core does not exceed the NS critical mass and does not collapse into a $\mathrm{BH}$, but still remains as a MNS with some additional orbiting material to guarantee the angular momentum conservation. As a consequence, no $\mathrm{GeV}$ emission is expected from these systems and, indeed, is not observed. The S-GRF occurrence rate is $\rho_{\mathrm{S}-\mathrm{GRF}}=3.6_{-1.0}^{+1.4} \mathrm{Gpc}^{-3} \mathrm{y}^{-1}$ [42] (see figure 1).

- Authentic short GRBs (S-GRBs) with $E_{\text {iso }} \gtrsim 10^{52} \mathrm{erg}$ and $E_{p, i} \gtrsim 2 \mathrm{MeV}$. They occur when a $\mathrm{BH}$ is formed in the NS-NS merger. Thus, these systems exhibit GeV emission. Their occurrence rate is $\rho_{\mathrm{S}-\mathrm{GRB}}=\left(1.9_{-1.1}^{+1.8}\right) \times$ $10^{-3} \mathrm{Gpc}^{-3} \mathrm{y}^{-1}$ [42] (see figure 1).

We have recently proposed the existence of ultra-short GRBs (U-GRBs), a new hybrid sub-class of (yet unobserved) short bursts originating from the BdHNe out-states ( $v$ NS-BH binaries), which remain bound nearly in $100 \%$ of the cases [48]. These systems represent a yet unaccounted family of merging NS-BH binaries in the current standard population synthesis analyses [48], therefore, including other possible channels of formation for NS-BH binaries, the lower limit of the U-GRB occurrence rate can be assumed equal to the $\mathrm{BdHN}$ rate, i.e., $\rho_{\mathrm{U}-\mathrm{GRB}} \gtrsim 0.77_{-0.08}^{+0.09} \mathrm{Gpc}^{-3} \mathrm{yr}^{-1}$ [42] (see figure 1).

Finally, we proposed another sub-class of sources originating in NS-WD mergers: gamma-ray flashes (GRFs), a class of long GRBs occurring in a CBM environment with low density, e.g., $\sim 10^{-3} \mathrm{~cm}^{-3}$, typical of the halos of the GRB host galaxies [42, 52, 53], not associated with $\mathrm{SNe}$ [54], and characterized by the presence of a macronova emission in the optical afterglow [55]. GRFs have $10^{51} \lesssim$ $E_{\text {iso }} \lesssim 10^{52}$ erg and $0.2 \lesssim E_{p, i} \lesssim 2 \mathrm{MeV}$ and, therefore, the NS-WD merger forms a MNS and not a BH [42]. NS-WD binaries, are notoriously very common astrophysical systems [56] and their possible formation channels have been studied [57, 58]; as proposed in Ref. [42], another less likely but yet possible channel of formation is the merger of a NS-WD binary produced from an S-GRF. The GRF rate of occurrence is $\rho_{\mathrm{GRF}}=1.02_{-0.46}^{+0.71} \mathrm{Gpc}^{-3} \mathrm{y}^{-1}$ [42] (see figure 1).

In all the above systems, the $10^{52}$ erg limit is clearly a function of the yet unknown precise value of the NS critical mass. As already pointed out in Ref. [42] the direct observation of the separatrix energy between S-GRFs and S-GRBs, and also between BdHNe and XRFs, gives fundamental informations for the determination of the actual value of maximum NS mass and for the minimum mass of the newly-formed $\mathrm{BH}$.

In this paper we review the latest observational and theoretical results which led to above burst classification scheme. In Section 2, we briefly summarize the fireshell model. In Section 3, we describe the observational properties of XRFs and BdHNe and their interpretation within the IGC paradism. In Section 4, we focus on the S-GRBs and specially on the theoretical interpretation of their $\mathrm{GeV}$ emission. In Section 4, we summarize our Conclusions.

\section{The fireshell model}

Before going into details in the observational and theoretical description of XRFs, BdHNe, S-GRFs and S-GRBs, we briefly summarize the fireshell model which is at the basis for the above classification of all bursts.

In the fireshell model [6-8], the GRB emission originate from an optically thick $e^{+} e^{-}$plasma of total energy $E_{e^{+} e^{-}}^{\text {tot }}-$ the fireshell. Its expansion and self-acceleration is due to the gradual $e^{+} e^{-}$annihilation [59]. Even after engulfing the baryonic mass $M_{B}$ left over by the progenitor system, quantified by the baryon load $B=M_{B} c^{2} / E_{e^{+} e^{-}}^{\text {tot }}$ [60], the fireshell remains still optically thick and continues its self-acceleration up to ultrarelativistic velocities $[61,62]$. When the fireshell reaches the transparency condition, a first flash of radiation, the P-GRB, is emitted $[7,59,60]$. The spectrum of the P-GRB is determined by the geometry of the pair-creation region: in the case of the spherically symmetric dyadosphere, the P-GRB spectrum is generally described by a single thermal component 


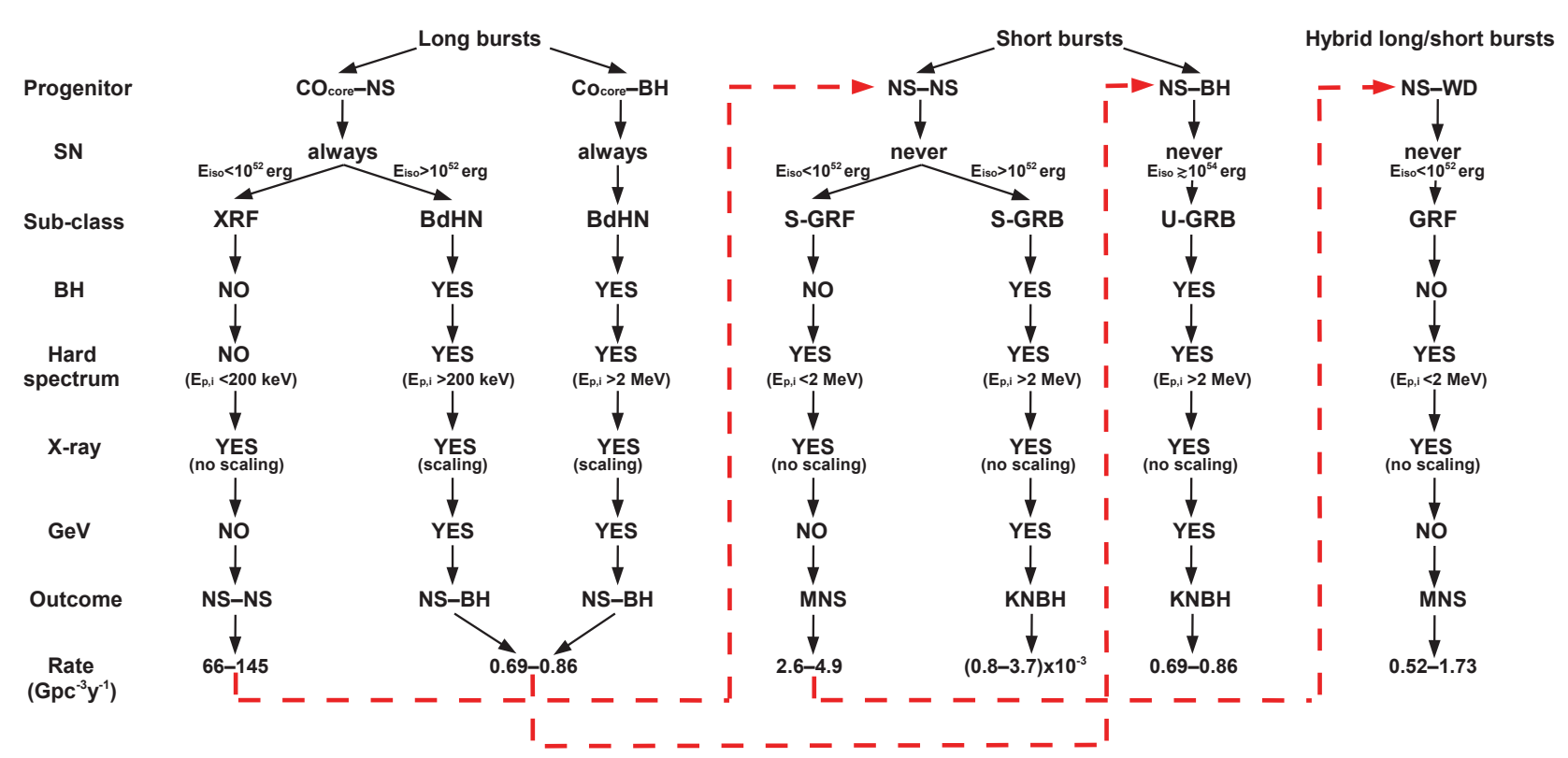

Figure 1. Summary of the properties of the burst sub-classes discussed in the Introduction. The red dashed lines indicate the evolutionary tracks linking out-states and in-states of some of the sub-classes. Additional details can be found in Ref. [42].

$[10,63]$; in the case of an axially symmetric dyadotorus, the resulting P-GRB spectrum is a convolution of thermal spectra of different temperatures which resembles more a power-law spectral energy distribution with an exponential cutoff $[64,65]$.

After transparency, the accelerated baryons propagate through and interact in fully radiative regime with the CBM, giving rise to the prompt emission [7]. The structures observed in the prompt emission of a GRB depend on the CBM density $n_{C B M}$ and its inhomogeneities [6668]. In both long and short bursts the CBM clouds have similar masses $\left(10^{22}-10^{24} \mathrm{~g}\right)$, sizes $\left(\approx 10^{15}-10^{16} \mathrm{~cm}\right)$, and typical distances from the $\mathrm{BH}\left(\approx 10^{16}-10^{17} \mathrm{~cm}\right)[10,33]$. The observed prompt emission spectrum results from the convolution of a large number of comoving spectra with decreasing temperatures and Lorentz and Doppler factors, due to each collision with the CBM, over the surfaces of constant arrival time for photons at the detector $[69,70]$ over the entire observation time.

To conclude, the evolution of an optically thick baryon-loaded pair plasma, is generally described in terms of $E_{e^{+} e^{-}}^{\text {tot }}$ and $B$ and it is independent of the way the pair plasma is created. This general formalism can also be applied to any optically thick $e^{+} e^{-}$plasma, like the one created via $v \bar{v} \leftrightarrow e^{+} e^{-}$mechanism in a NS merger as described in $[17,71,72]$.

\section{XRFs and BdHNe in the IGC paradigm}

We here focus on the comparison between XRF and BdHN sub-classes within the IGC paradigm, giving a special attention to the latest theoretical results on the BdHNe.

In the IGC scenario, both XRFs and BdHNe originate in the hypercritical accretion process of the SN ejecta onto the NS binary companion. In this phenomenon pho- tons are trapped in the accreting material and the accretion energy is lost through a large associated neutrino emission $[39,40,73,74]$. In the XRFs, the $\mathrm{CO}_{\text {core }}-\mathrm{NS}$ binary is widely separated $\left(a \gtrsim 10^{11} \mathrm{~cm}\right)$, thus the accretion rate $<10^{-2} M_{\odot} \mathrm{s}^{-1}$ can only push the binary companion NS to become a MNS. The resulting emission, dubbed Episode 1, lasts $\sim 10^{2}-10^{4} \mathrm{~s}$. Its spectrum is characterized by: 1) a thermal component spectrum with temperatures in the range of $0.1-2 \mathrm{keV}$ and corresponding radii of $10^{10}-10^{12} \mathrm{~cm}$ (see figure 2 , left-panel), possibly originating from the outflow within the NS atmosphere driven out by Rayleigh-Taylor convection instabilities [40], and 2) a power-law component, possibly related to the excess of angular momentum of the system which necessarily leads to a jetted emission [41]. The long lasting X-ray emission does not exhibit any specific common late power-law behavior (see figure 2, right panel) and can be explained by the emission of the SN ejecta shocked by the hypercritical accretion emission of the XRF. This energy injection into the SN ejecta leads to the occurrence of a broad-lined SN Ic [75] with a kinetic energy larger than that of the traditional SNe Ic [42]. The absence of $\mathrm{GeV}$ emissions is implicit in the nature of the hypercritical accretion process not leading to a BH. Of course, all XRFs at redshift $z \lesssim 1$ exhibit an optical SN with a luminosity similar to the one of SN 1998bw [76], which occurs after 10-15 days in the source cosmological rest-frame.

In the IGC paradigm, the shorter the $\mathrm{CO}_{\text {core }}$-NS binary period, the larger the accretion rate and the values of $E_{\text {iso }}$ and $E_{\mathrm{p}, \mathrm{i}}$, and correspondingly the shorter the prompt emission duration [41]. Indeed, in $\mathrm{BdHNe}$ the $\mathrm{CO}_{\text {core }}-\mathrm{NS}$ binary is more tightly bound $\left(a \lesssim 10^{11} \mathrm{~cm}\right)$ and the accretion rates of the SN ejecta can be $\gtrsim 10^{-2}-10^{-1} M_{\odot} \mathrm{s}^{-1}$, leading the companion NS to collapse to a $\mathrm{BH}[40,41]$. For 

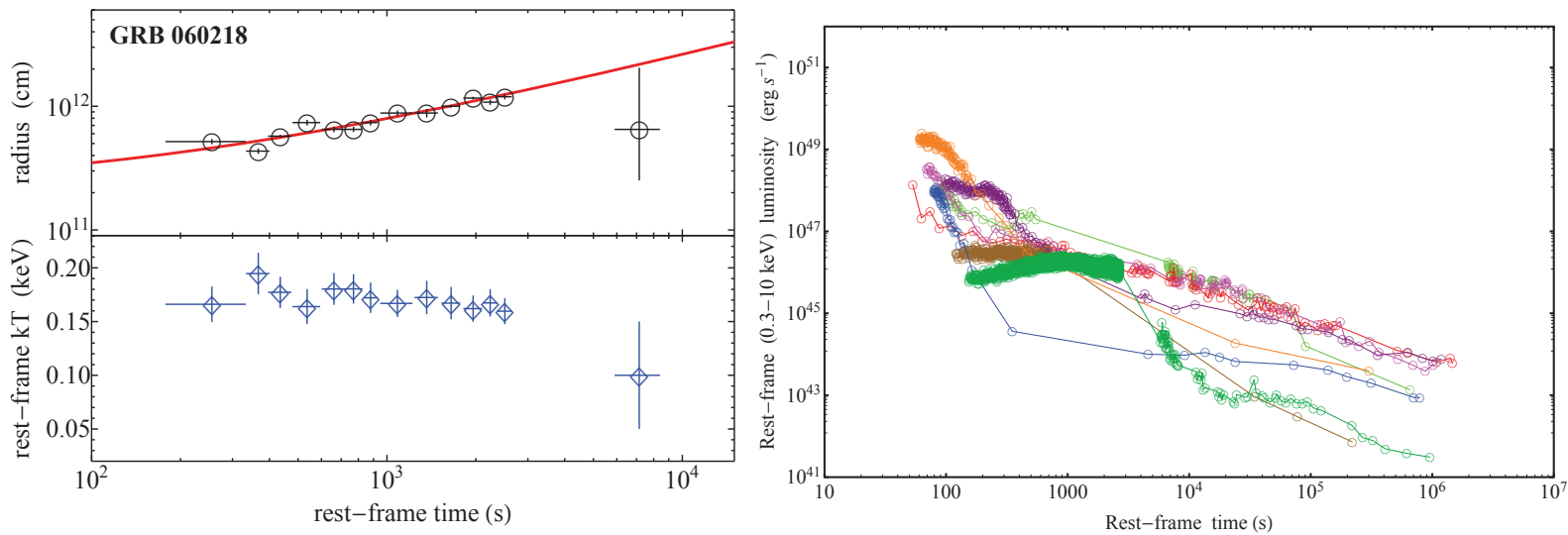

Figure 2. Left panel: the evolution of the radius of the thermal component detected in GRB 060218 (black circles) and its linear fit (solid red curve) and of the corresponding rest-frame temperature (blue diamonds). Reproduced from Ref. [77]. Right panel: restframe X-ray 0.3-10 keV luminosity light curves of selected XRFs: 050416A (red), 060218 (dark green), 070419A (orange), 081007 (magenta), 100316D (brown), 101219B (purple), and 130831A (green).

this reason BdHNe exhibit a more complex structure than XRFs composed of distinct Episodes.

- Episode 1 in BdHNe, like in the case of XRFs, also originates in the hypercritical accretion process. The corresponding spectrum again exhibits: 1) an expanding thermal component with a decreasing temperature, typical radii of $10^{9}-10^{10} \mathrm{~cm}$ and an average expansion speed of $\sim 10^{8}-10^{9} \mathrm{~cm} \mathrm{~s}^{-1}$ (see figure $3(\mathrm{a})$ ), and b) a power-law function $[33,34,78]$.

- Episode 2 corresponds to the $\gamma$-ray prompt emission of an authentic long GRB (see figure 3 (b)), stemming from the collapse of the companion NS to a BH and leading to the vacuum polarization process and the creation of an $e^{+} e^{-}$plasma. The analysis of the P-GRB emission indicates that BdHNe have a baryon load of $B \approx 10^{-4}$ $10^{-2}$ and at transparency they reach a Lorentz factor of $\Gamma=10^{2}-10^{3}$. The prompt emission is produced by the interaction of the fireshell with the CBM clouds located at $\sim 10^{16}-10^{17} \mathrm{~cm}$ from the burst site with average number density of $\sim 1 \mathrm{~cm}^{-3}[33,78]$.

- Episode 3 occurs after the prompt emission in the Xrays. It composed of a steep decay characterized by the presence of an early X-ray flare, a plateau and a late power-law decay which we refer as to the afterglow. These three components are dubbed flare-plateauafterglow (FPA) phase [79]. During the early X-ray flare phase (typically at a rest-frame time of $\sim 10^{2} \mathrm{~s}$ ) an expanding thermal component has been observed in its spectrum $[9,47,79]$. The inferred radii are typically $\sim 10^{12}-10^{13} \mathrm{~cm}$ and they expand at mildly relativistic speed with $\Gamma \lesssim 4[9,47,78,79]$. The size of the corresponding emitting region is clearly incompatible with the radii inferred from Episodes 1 and 2. When computed in the source cosmological rest-frame, the plateau and the late power-law decay exhibit new features (see figure 3 (c)): 1) the overlapping of the afterglow phases, which have typical slopes of $-1.7 \lesssim \alpha \lesssim-1.3$ and show a characteristic common power-law behavior [46]; the nested property, which shows that the duration (the lu- minosity) of the plateau phase is inversely (directly) proportional to the energy of the GRB emission, i.e., the more energetic the source, the smaller (higher) the duration (the luminosity) of the plateau [47]. The use of the overlapping of the afterglows as a distance indicator has been explored by inferring the redshifts of GRB 101023 [34], and has been applied to predict the occurrence of the SN associated to GRB 130427A before its discovery [80], later confirmed by the observations [8184]. In the IGC scenario, the FPA originates from the SN ejecta $[9,79]$. In BdHNe the SN ejecta experiences an energy injection from GRB emission leading to the occurrence of a broad-lined SN Ic [75] with a kinetic energy larger than that of the traditional SNe Ic. This energy injection results in an isotropic energy emission of $10^{51}-10^{52}$ erg for the FPA phase. In particular, the $\mathrm{X}$-ray flare can be modeled by considering the impact of the GRB on the SN ejecta and the propagation of the optically thick $e^{+} e^{-}$plasma into a medium largely baryon-contaminated $\left(B \approx 10-10^{2}\right)$. A numerical integration starting at $10^{10} \mathrm{~cm}$ all the way to $10^{12} \mathrm{~cm}$, where the transparency is reached, gives a perfect agreement between the radius of the emitter at transparency and the observations, as well a coincidence of the observed time of the peak emission of the flare [79]. The plateau and the afterglow phases are still under study (M. Karlica et al., in preparation).

- Episode 4 corresponds to the optical SN emission observable in all BdHNe at $z \lesssim 1$ after $\approx 10-15$ days in the cosmological rest-frame. All these $\mathrm{SNe}$ have a standard luminosity similar to the one of SN 1998bw [76].

- Episode 5 is identified with the distinctive long-lived $\mathrm{GeV}$ emission, observed in the majority of BdHNe when within the LAT field of view. Though this emission follows a precise power-law behavior with index $\approx-1.2$ $[42,85]$ (see figure $3(d)$ ), this emission is conceptually distinct in its underlying physical process from that of Episode 3: it originate, in facts, in the further accretion of matter onto the newly-formed $\mathrm{BH}$ and it is ob- 
servable only after the transparency emission, i.e., the P-GRB [42].

\section{The S-GRBs in the NS-NS merger paradigm}

In Section 1 we discussed the rates of S-GRFs and SGRBs, showing that S-GRFs are the most frequent events among the short bursts. This result is also in good agreement with the NS-NS binaries observed within our Galaxy: only a subset of them has a total mass larger than the NS critical mass $M_{\text {crit }}^{\mathrm{NS}}$ and can form a BH in their merging process [10] if we assume that $M_{\text {crit }}^{\mathrm{NS}}=2.67 M_{\odot}$ for a non-rotating, globally neutral NS within the NL3 nuclear model [87]. In this light S-GRBs are very important for inferring constraints on $M_{\text {crit }}^{\mathrm{NS}}$, on the NS equation of state, and on the minimum mass of the newly-formed $\mathrm{BH}$.

To date, within the fireshell model we have analized five authentic S-GRBs: 090227B [63], 140619B [10], GRB 090510 [64], 081024B and 140402A [65]. The analyses of the P-GRB emission and the correlation between the spikes of the prompt emission and CBM inhomogeneities gave the most successful test for the fireshell model. S-GRBs share some remarkable analogies but also some differences with BdHNe in view of the simplicity of the underlying physical system of S-GRBs, which unlike the BdHNe, do not exhibit any of the extremely complex activities related to the $\mathrm{SN}$ (see Section 3).

- Episode 1 here is related to the NS-NS merger activity before the gravitational collapse into a $\mathrm{BH}$ and possibly corresponds to faint precursors observed in some short bursts $[64,88]$. Because of the compactness of the systems this process at times is not observable.

- Episode 2 corresponds to the GRB emission from the NS-NS merger. Within the fireshell model it is composed of the P-GRB, which occur before the onset of the $\mathrm{GeV}$ emission, and the prompt emission (see figure 4, left panel). From the analysis of their P-GRB emission, all S-GRBs have a standard values of the baryon load $\left(B \approx 5 \times 10^{-5}\right)$, which is consistent with the crustal masses of NS-NS mergers [10, 89], and of the Lorentz factors at the transparency $\Gamma \approx 10^{4}[10,63-65]$. From the fit of the prompt emission (see figure 4, right panel), it came out that S-GRBs occur in a standard CBM with average density $\left\langle n_{\mathrm{CBM}}\right\rangle \approx 10^{-5} \mathrm{~cm}^{-3}$ [10, 63-65], which is typical of galactic halos where NS-NS mergers migrate, owing to natal kicks imparted to the binaries at birth [22].

- Episode 3, which corresponds to the traditional X-ray afterglow, differs from that of BdHNe which results from the interaction between the GRB and the SN ejecta. Work on this topic is still ongoing.

- Episode 4, identified with the optical emission of a SN, is here missing.

- Episode 5 coincides with the $\mathrm{GeV}$ emission turning on soon after the P-GRB and being coeval with the prompt emission. With the exception of GRB 090227B, which was outside the nominal Fermi-LAT field of view [86], all S-GRBs consistently exhibit this emission, which appears to be strictly correlated to that observed in the $\mathrm{BdHNe}$. Since the presence of a $\mathrm{BH}$ is the only commonality between BdHNe and S-GRBs, by analogy we assume that the $\mathrm{GeV}$ emission originate from the activity of the newly-born BH produced in the merger [10]. The rest-frame $0.1-100 \mathrm{GeV}$ luminosity light curves of all S-GRBs with LAT data follow a common powerlaw behavior with the rest-frame time which goes as $t^{-1.29 \pm 0.06}$ (see dashed black line in figure 5).

Table 1 lists the redshift, $E_{p, i}, E_{i s o}$ (in the rest-frame energy band $1-10000 \mathrm{keV}$ ), and the $\mathrm{GeV}$ isotropic emission energy $E_{L A T}$ (in the rest-frame energy band 0.1-100 $\mathrm{GeV}$ ) of all S-GRBs. The values of $E_{L A T}$ represent lower limits to the actual $\mathrm{GeV}$ isotropic emission energies, since at late times the observations of $\mathrm{GeV}$ emission could be prevented due to instrumental threshold of the LAT. Using the maximum $\mathrm{GeV}$ photon observed energy $E_{\mathrm{GeV}}^{\max }$ listed in table 1, we derive a lower limit on the Lorentz factor of the $\mathrm{GeV}$ emission $\Gamma_{\mathrm{GeV}}^{\min }$ by requiring the optically thin condition to the high energy photons [90]. For each S-GRB we estimate lower limits in each time interval of the $\mathrm{GeV}$ luminosity light curves in figure 5 . Then, $\Gamma_{\mathrm{GeV}}^{\mathrm{min}}$ for each $\mathrm{S}-\mathrm{GRB}$ has been then determined as the largest among the inferred lower limits. It follows that the $\mathrm{GeV}$ emission is produced by an ultrarelativistic outflows with $\Gamma_{\mathrm{GeV}}^{\min } \gtrsim 300$ (see table 1).

We propose that the $\mathrm{GeV}$ emission in S-GRBs is produced by accretion onto the new-born $\mathrm{BH}$ of a certain amount of mass that remains bound to it because of the conservation of energy and angular momentum from the merger moment to the BH birth [64]. Lower limits on the amount of accreted mass can be attained by considering the accretion process onto a maximally rotating Kerr $\mathrm{BH}$. Depending whether the infalling material is in co- or counter-rotating orbit with the spinning $\mathrm{BH}$, the maximum efficiency of the conversion of gravitational energy into radiation is $\eta_{+}=42.3 \%$ or $\eta_{-}=3.8 \%$, respectively [91] and, therefore, $E_{\mathrm{LAT}}$ can be expressed as

$$
E_{\mathrm{LAT}}=f_{\mathrm{b}}^{-1} \eta_{ \pm} M_{\mathrm{acc}}^{\eta_{ \pm}} c^{2},
$$

where $f_{\mathrm{b}}$ is the beaming factor which depends on the geometry of the GeV emission, and $M_{\mathrm{acc}}^{\eta_{ \pm}}$is the amount of accreted mass depending on the choice of the efficiency. The observational evidence that the totality of S-GRBs exhibit $\mathrm{GeV}$ emission and that its absence is due instrumental absence of alignment between the LAT and the source at the time of the GRB emission suggest no significant beaming. Therefore, in the following we set $f_{\mathrm{b}} \equiv 1$. The corresponding estimates of $M_{\mathrm{acc}}^{\eta_{ \pm}}$in our sample of S-GRBs are listed in table 1 .

\section{Conclusions}

Remarkable progresses in the understanding of GRBs have been made possible thanks to the great amount X- and $\gamma$ rays and high energy data and to a deeper theoretical understanding of WD [92], NS [87, 89] and BH [93], leading to a new paradigm purporting the role of binary systems as progenitors of GRBs: $\mathrm{CO}_{\text {core }}-\mathrm{NS}$ binaries for long 


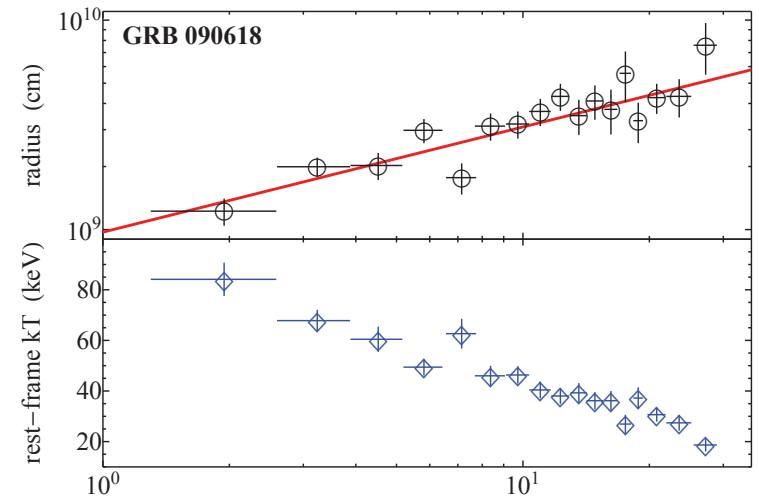

(a)

rest-frame time (s)

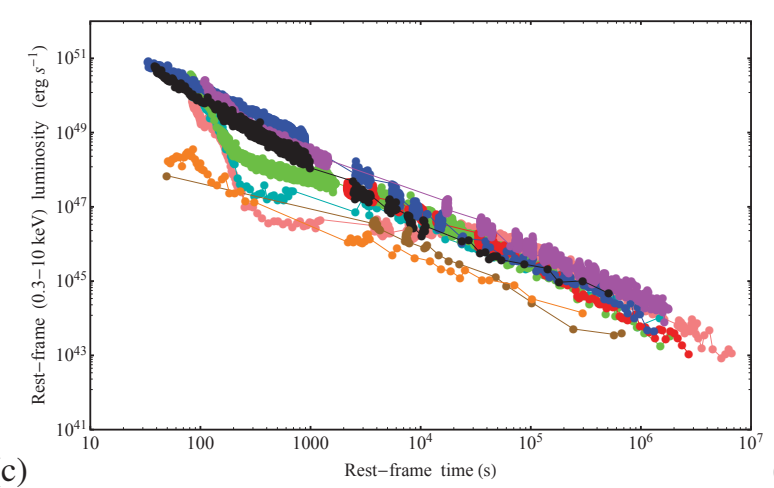

(d)

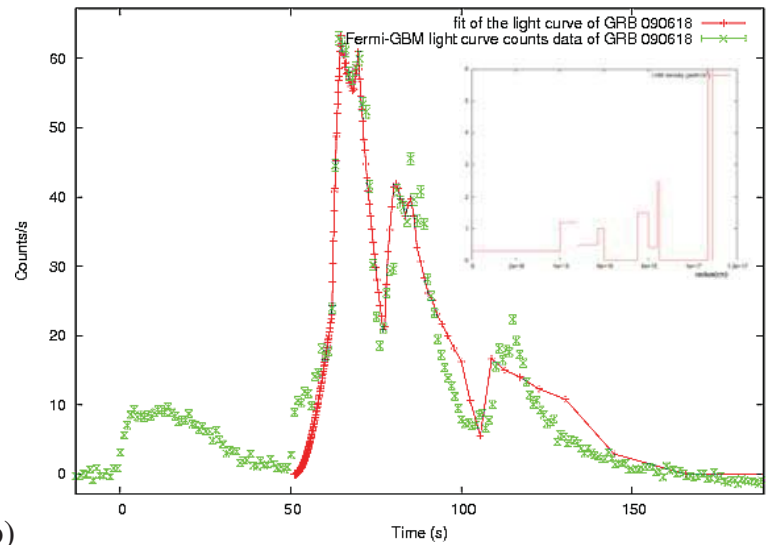

(b)

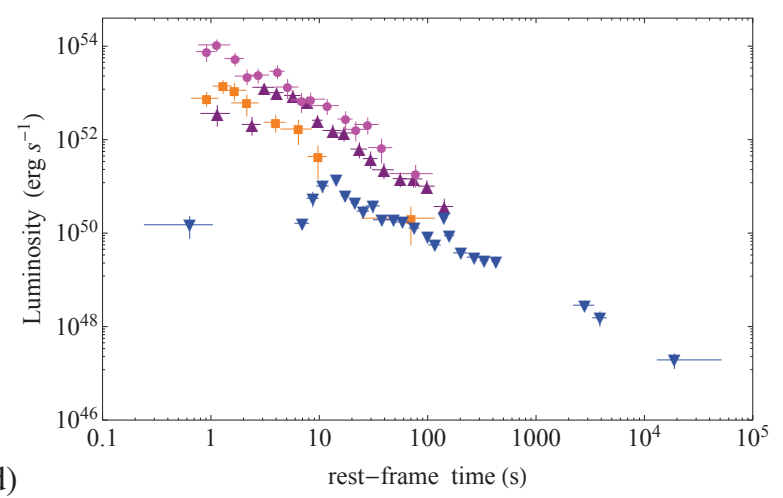

Figure 3. (a) The evolution of the radius of the thermal component detected in the Episode 1 of GRB 090618 (black circles) and its linear fit (solid red curve), and the decay of the corresponding rest-frame temperature (blue diamonds). (b) The fireshell simulation (red line) of the light curve of Episode 2 of the prototype GRB 090618 (green data). The small inset reproduces the CBM profile required for the simulation. Reproduced from Ref. [33]. (c) The rest-frame 0.3-10 keV luminosity light curves of selected BdHNe: 050525 (brown), 060729 (pink), 061007 (black), 080319B (blue), 090618 (green), 091127 (red), 100816A (orange), 111228A (light blue), and 130427A (purple) [79]. (d) The rest-frame 0.1-100 GeV luminosity light curves [86] of selected BdHNe: GRB 080916C (magenta circles), GRB 090902B (purple triangles), GRB 110731A (orange squares), GRB 130427A (blue reversed triangles).
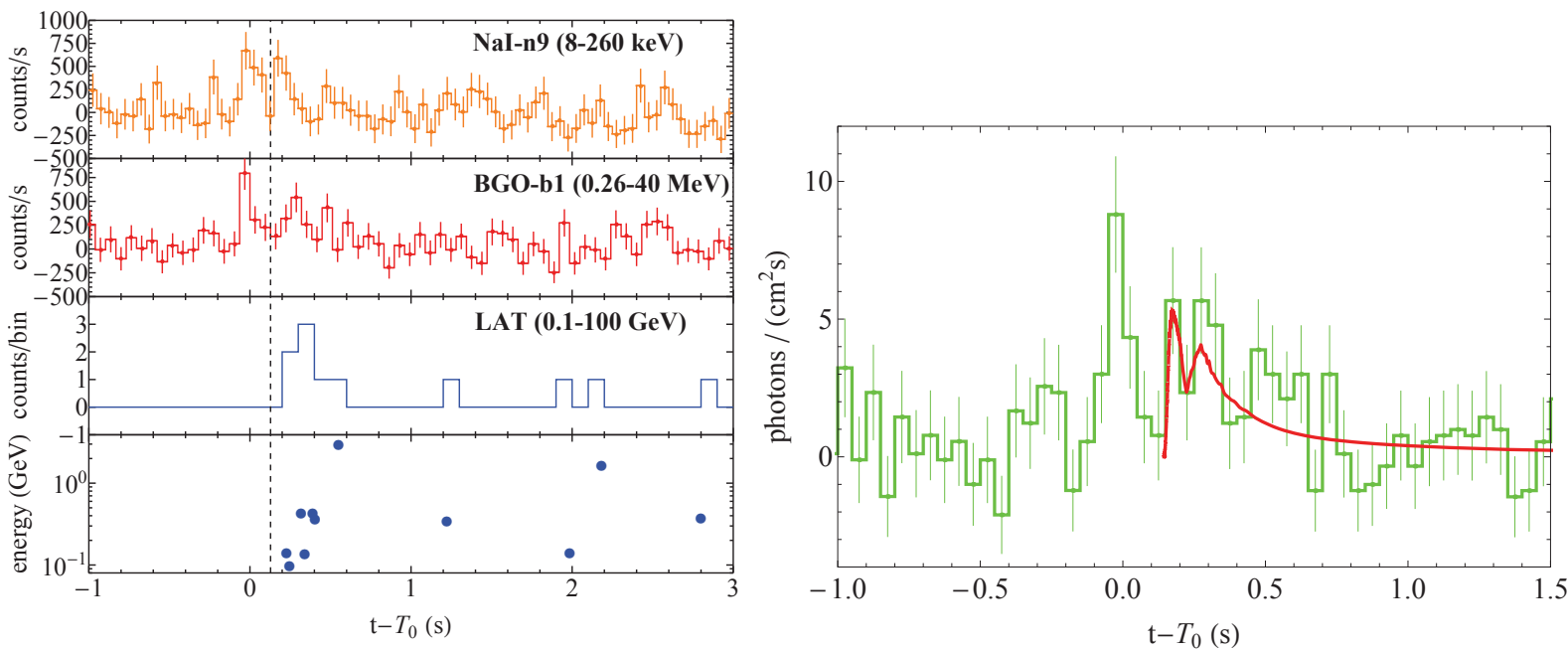

Figure 4. Left panel: background subtracted $50 \mathrm{~ms}$ binned data from the NaI-n9 (8 - $260 \mathrm{keV}$, top panel) and BGO-b1 (0.26- $40 \mathrm{MeV}$, second panel) detectors, the $100 \mathrm{~ms}$ binned high energy data $(0.1-100 \mathrm{GeV}$, third panel, without error bars), and the high energy photons detected by the of the Fermi-LAT (bottom panel) for the S-GRB 081024B; the vertical dashed line marks the on-set of the LAT light curve. Right panel: the NaI-n9 light curve of the prompt emission of the S-GRB 081024B (green data) and the simulation within the fireshell model (red curve). All plots are reproduced from Ref. [65]. 


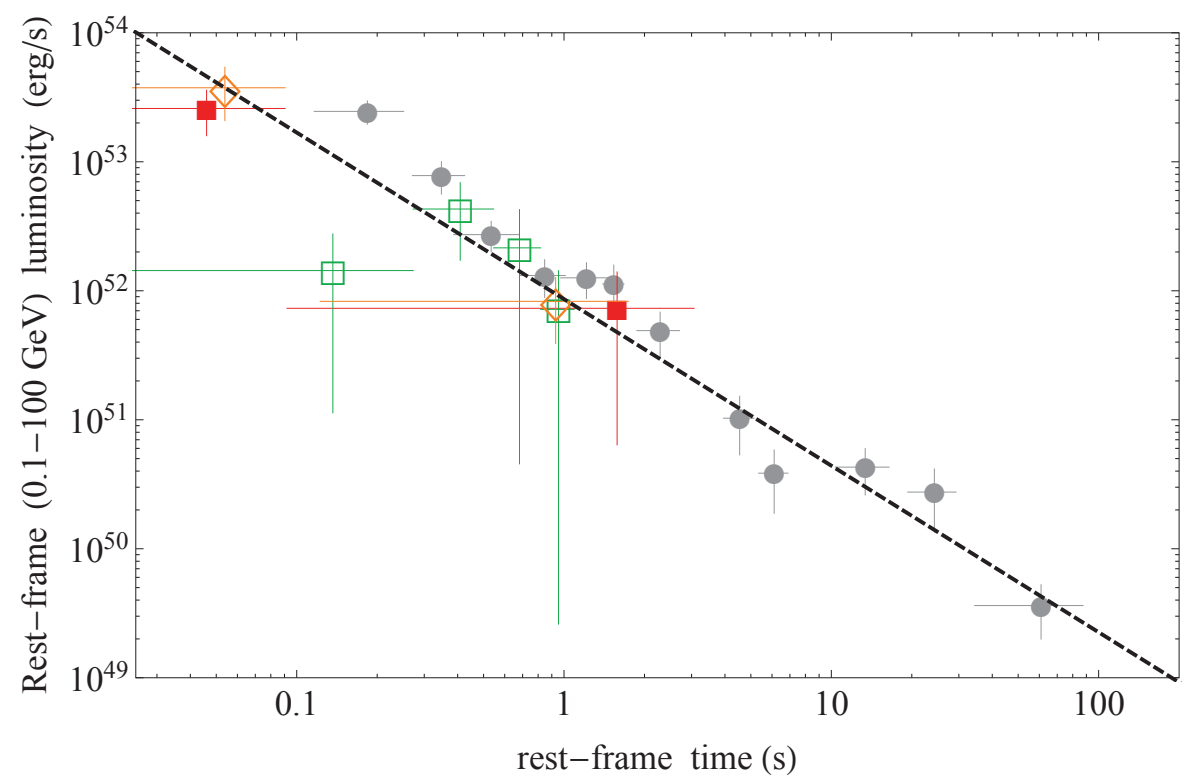

Figure 5. The rest-frame 0.1-100 GeV isotropic luminosities of the S-GRBs: 081024B (orange empty diamonds), 090510 (gray filled circles), 140402A (red filled squares), and 140619B (green empty squares). All the light curves start after the P-GRB emission. The dashed black line marks the common behavior of all the S-GRB light curves which goes as $t^{-1.29 \pm 0.06}$. Reproduced from Ref. [65].

\begin{tabular}{lcccccccc}
\hline \hline GRB & $\mathrm{z}$ & $\begin{array}{c}E_{\mathrm{p}, \mathrm{i}} \\
(\mathrm{MeV})\end{array}$ & $\begin{array}{c}E_{\mathrm{iso}} \\
\left(10^{52} \mathrm{erg}\right)\end{array}$ & $\begin{array}{c}E_{\mathrm{GeV}}^{\mathrm{max}} \\
(\mathrm{GeV})\end{array}$ & $\Gamma_{\mathrm{GeV}}^{\min }$ & $\begin{array}{c}E_{\mathrm{LAT}} \\
\left(10^{52} \mathrm{erg}\right)\end{array}$ & $\begin{array}{c}M_{\mathrm{acc}}^{\eta_{+}} \\
\left(\mathrm{M}_{\odot}\right)\end{array}$ & $\begin{array}{c}M_{\mathrm{acc}}^{\eta_{-}} \\
\left(\mathrm{M}_{\odot}\right)\end{array}$ \\
\hline 081024B & $3.12 \pm 1.82$ & $9.56 \pm 4.94$ & $2.64 \pm 1.00$ & 3 & $\gtrsim 779$ & $\gtrsim 2.79 \pm 0.98$ & $\gtrsim 0.04$ & $\gtrsim 0.41$ \\
$090227 \mathrm{~B}$ & $1.61 \pm 0.14$ & $5.89 \pm 0.30$ & $28.3 \pm 1.5$ & - & - & - & - & - \\
090510 & $0.903 \pm 0.003$ & $7.89 \pm 0.76$ & $3.95 \pm 0.21$ & 31 & $\gtrsim 551$ & $\gtrsim 5.78 \pm 0.60$ & $\gtrsim 0.08$ & $\gtrsim 0.86$ \\
$140402 \mathrm{~A}$ & $5.52 \pm 0.93$ & $6.1 \pm 1.6$ & $4.7 \pm 1.1$ & 3.7 & $\gtrsim 354$ & $\gtrsim 4.5 \pm 2.2$ & $\gtrsim 0.06$ & $\gtrsim 0.66$ \\
$140619 \mathrm{~B}$ & $2.67 \pm 0.37$ & $5.34 \pm 0.79$ & $6.03 \pm 0.79$ & 24 & $\gtrsim 471$ & $\gtrsim 2.34 \pm 0.91$ & $\gtrsim 0.03$ & $\gtrsim 0.35$ \\
\hline
\end{tabular}

Table 1. S-GRB properties: $z, E_{\mathrm{p}, \mathrm{i}}$, the maximum $\mathrm{GeV}$ photon observed energy $E_{\mathrm{GeV}}^{\max }$, the minimum Lorentz factor of the $\mathrm{GeV}$ emission $\Gamma_{\mathrm{GeV}}^{\mathrm{min}}, E_{i s o}, E_{\mathrm{LAT}}$, and the amount of infalling accreting mass co-rotating (counter-rotating) with the $\mathrm{BH} M_{\mathrm{acc}}^{\eta_{+}}\left(M_{\mathrm{acc}}^{\eta_{-}}\right)$, needed to explaing $E_{\mathrm{LAT}}$.

bursts within the IGC paradigm [6, 38-40], and NS-NS (or NS-BH) binaries for short bursts, as widely accepted and confirmed by strong observational and theoretical evidences [12-22]. These paradigms have led to the classification of GRBs in seven different sub-classes (see figure 1). We here focus our attention on the sub-classes of XRFS, BdHNe, S-GRFs and S-GRBs.

In Section 2, we review the fireshell model for GRBs [6-8] and its essential role in order to disentangle the various emission episodes characterizing each of the above sub-classes.

In Section 3, we summarize the commonalities and the differences between the observational properties of XRFs and $\mathrm{BdHNe}$ and provide their theoretical interpretation within the IGC paradigm, namely, whether or not the hypercritical accretion process leads to the formation of a $\mathrm{BH}$.

In Section 4, we outline the properties S-GRFs and SGRBs originating in NS-NS mergers leading to a MNS and the formation of a $\mathrm{BH}$, respectively. Then, we focus on S-GRBs and on the key role of the P-GRB identification for their description, as well as the analysis of the $\mathrm{GeV}$ emission. We finally discuss in details the $\mathrm{GeV}$ emission uniquely observed in both BdHNe and S-GRBs, when within the Fermi-LAT FoV. In both cases it starts after the P-GRB emission and it is coeval with the occurrence of the prompt emission [42]. Moreover, the restframe 0.1-100 GeV luminosities in BdHNe and S-GRBs share a common luminosity pattern, a precise power-law behavior with time $\propto t^{-1.2}[9,10,42,65,85]$. These commonalities, in such different systems, as well as their energy requirements are naturally explained if we assume that the $\mathrm{GeV}$ emission originates by accretion processes in the newly-born $\mathrm{BH}[9,10]$. In all the identified S-GRBs, within the Fermi-LAT FoV, GeV photons are always observed $[42,64]$. This implies that no intrinsic beaming is necessary to explain the S-GRB GeV emission. Within the hypothesis of isotropic emission, in the case of S-GRBs we point out how the total energy of the GeV emission can attained from the gravitational binding energy of mass accretion of $M \gtrsim 0.03-0.08 M_{\odot}$ or $M \gtrsim 0.35-0.86 M_{\odot}$ for coor counter-rotating orbits with a maximally rotating $\mathrm{BH}$, respectively (see table 1). A lower limit on the Lorentz factor of the $\mathrm{GeV}$ emission of $\Gamma_{\mathrm{GeV}}^{\mathrm{min}} \gtrsim 300$ can be obtained 
by requiring the optically thin condition to the high energy photons [90].

From the above consideration and the proposed classification scheme some considerations follow.

- The knowledge of the separatrix energy of $10^{52} \mathrm{erg}$, which discriminates between systems forming or not BHs and on which our classification scheme of GRBs is based, represents an observational constraints on the value of the NS critical mass $M_{\text {crit }}$, certainly in the range of 2.2-2.7 $M_{\odot}$ for a non-rotating NS depending on the equations of state [87], and the minimum mass of a $\mathrm{BH}$. This value is consistent and can be derived in BdHNe by considering the hypercritical accretion process onto a NS leading to an energy release in form of neutrinos and photons, given by the gain of gravitational potential energy of the matter accreted in the NS. This includes the change of binding energy of the NS while accreting both matter and angular momentum [42].

- Most noteworthy, the existence of a precise common power-law behavior in the rest-frame $0.1-100 \mathrm{GeV}$ luminosities of S-GRBs (see figure 5), following the $\mathrm{BH}$ formation, points to a commonality in the mass and spin of the newly-formed $\mathrm{BH}$. This result is explainable with the expected mass of the merging NSs, each one of mass $M \approx 1.3-1.5 M_{\odot}$ [94], and the above expected range of the non-rotating NS critical mass, leading to a standard value of the $\mathrm{BH}$ mass and of its Kerr parameter [10].

- Finally, we discuss the gravitational wave $(\mathrm{GW})$ detectability by advanced LIGO (aLIGO) from S-GRBs. We have already shown that binaries in which each NS has a mass $M_{\mathrm{NS}}=1.34 M_{\odot}=0.5 M_{\text {crit }}^{\mathrm{NS}}$ produce $\mathrm{GW}$ signals which are well below the signal to noise ratio $\mathrm{S} / \mathrm{N}=8$ needed for a positive detection by aLIGO: a positive GW detection may occur only for sources located at $z \lesssim 0.14$ for the aLIGO 2022+ run, a redshift well above that of GRB 090510, to date the closest S-GRB located at $z=0.903[10,95,96]$.

M. M. and J. A. R. acknowledge the partial support of the project N 3101/GF4 IPC-11, and the target program F.0679 0073-6/PTsF of the Ministry of Education and Science of the Republic of Kazakhstan.

\section{References}

[1] E.P. Mazets, S.V. Golenetskii, V.N. Ilinskii, V.N. Panov, R.L. Aptekar, I.A. Gurian, M.P. Proskura, I.A. Sokolov, Z.I. Sokolova, T.V. Kharitonova, Astrophysics and Space Science 80, 3 (1981)

[2] J.P. Dezalay, C. Barat, R. Talon, R. Syunyaev, O. Terekhov, A. Kuznetsov, Short cosmic events A subset of classical GRBs?, in American Institute of Physics Conference Series, edited by W. S. Paciesas \& G. J. Fishman (1992), Vol. 265 of American Institute of Physics Conference Series, pp. 304-309

[3] R.W. Klebesadel, The durations of gamma-ray bursts, in Gamma-Ray Bursts - Observations, Analyses and Theories, edited by C. Ho, R.I. Epstein, E.E. Fenimore (Cambridge University Press, 1992), pp. $161-168$
[4] C. Kouveliotou, C.A. Meegan, G.J. Fishman, N.P. Bhat, M.S. Briggs, T.M. Koshut, W.S. Paciesas, G.N. Pendleton, ApJ Letters 413, L101 (1993)

[5] M. Tavani, ApJ Letters 497, L21 (1998), arXiv: astro-ph/9802192

[6] R. Ruffini, C.L. Bianco, F. Fraschetti, S.S. Xue, P. Chardonnet, ApJ Letters 555, L117 (2001), arXiv:astro-ph/0106534

[7] R. Ruffini, C.L. Bianco, F. Fraschetti, S.S. Xue, P. Chardonnet, ApJ Letters 555, L113 (2001), arXiv: astro-ph/0106532

[8] R. Ruffini, C.L. Bianco, F. Fraschetti, S.S. Xue, P. Chardonnet, ApJ Letters 555, L107 (2001), arXiv:astro-ph/0106531

[9] R. Ruffini, Y. Wang, M. Enderli, M. Muccino, M. Kovacevic, C.L. Bianco, A.V. Penacchioni, G.B. Pisani, J.A. Rueda, ApJ 798, 10 (2015), 1405. 5723

[10] R. Ruffini, M. Muccino, M. Kovacevic, F.G. Oliveira, J.A. Rueda, C.L. Bianco, M. Enderli, A.V. Penacchioni, G.B. Pisani, Y. Wang et al., ApJ 808, 190 (2015), 1412.1018

[11] R. Ruffini, J.A. Rueda, M. Muccino, G.B. Pisani, Y. Wang, L.M. Becerra, M. Kovacevic, F.G. Oliveira, Y. Aimuratov, C.L. Bianco et al., ArXiv e-prints (2016), 1602.02732

[12] J. Goodman, ApJ Letters 308, L47 (1986)

[13] B. Paczynski, ApJ Letters 308, L43 (1986)

[14] D. Eichler, M. Livio, T. Piran, D.N. Schramm, Nature 340, 126 (1989)

[15] R. Narayan, T. Piran, A. Shemi, ApJ Letters 379, L17 (1991)

[16] P. Meszaros, M.J. Rees, ApJ Letters 482, L29 (1997), astro-ph/9609065

[17] S. Rosswog, E. Ramirez-Ruiz, M.B. Davies, MNRAS 345, 1077 (2003), astro-ph/0110180

[18] W.H. Lee, E. Ramirez-Ruiz, D. Page, ApJ Letters 608, L5 (2004), astro-ph/0404566

[19] E. Nakar, Physics Reports 442, 166 (2007), astro-ph/0701748

[20] A. Endrizzi, R. Ciolfi, B. Giacomazzo, W. Kastaun, T. Kawamura, ArXiv e-prints (2016), 1604.03445

[21] M. Ruiz, R.N. Lang, V. Paschalidis, S.L. Shapiro, ApJ Letters 824, L6 (2016), 1604 . 02455

[22] E. Berger, Annual Review of A\&A 52, 43 (2014), 1311.2603

[23] K.C. Sahu, M. Livio, L. Petro, F.D. Macchetto, J. van Paradijs, C. Kouveliotou, G.J. Fishman, C.A. Meegan, P.J. Groot, T. Galama, Nature 387, 476 (1997), arXiv: astro-ph/9705184

[24] J. van Paradijs, P.J. Groot, T. Galama, C. Kouveliotou, R.G. Strom, J. Telting, R.G.M. Rutten, G.J. Fishman, C.A. Meegan, M. Pettini et al., Nature 386, 686 (1997)

[25] J.S. Bloom, J.X. Prochaska, D. Pooley, C.H. Blake, R.J. Foley, S. Jha, E. Ramirez-Ruiz, J. Granot, A.V. Filippenko, S. Sigurdsson et al., ApJ 638, 354 (2006), arXiv: astro-ph/0505480 
[26] E. Troja, A.R. King, P.T. O’Brien, N. Lyons, G. Cusumano, MNRAS 385, L10 (2008), 0711.3034

[27] W. Fong, E. Berger, D.B. Fox, ApJ 708, 9 (2010), 0909. 1804

[28] E. Berger, New Astron. Rev. 55, 1 (2011), 1005.1068

[29] D. Kopač, P. D’Avanzo, A. Melandri, S. Campana, A. Gomboc, J. Japelj, M.G. Bernardini, S. Covino, S.D. Vergani, R. Salvaterra et al., MNRAS 424, 2392 (2012), 1203. 1864

[30] S.E. Woosley, J.S. Bloom, Annual Review of A\&A 44, 507 (2006), astro-ph/0609142

[31] A.S. Fruchter, A.J. Levan, L. Strolger, P.M. Vreeswijk, S.E. Thorsett, D. Bersier, I. Burud, J.M. Castro Cerón, A.J. Castro-Tirado, C. Conselice et al., Nature 441, 463 (2006), arXiv:astro-ph/0603537

[32] K.M. Svensson, A.J. Levan, N.R. Tanvir, A.S. Fruchter, L.G. Strolger, MNRAS 405, 57 (2010), 1001.5042

[33] L. Izzo, R. Ruffini, A.V. Penacchioni, C.L. Bianco, L. Caito, S.K. Chakrabarti, J.A. Rueda, A. Nandi, B. Patricelli, A\&A 543, A10 (2012), 1202.4374

[34] A.V. Penacchioni, R. Ruffini, L. Izzo, M. Muccino, C.L. Bianco, L. Caito, B. Patricelli, L. Amati, A\&A 538, A58 (2012), 1112.2970

[35] N. Smith, Annual Review of A\&A 52, 487 (2014), 1402.1237

[36] N. Smith, W. Li, A.V. Filippenko, R. Chornock, MNRAS 412, 1522 (2011), 1006.3899

[37] M. Della Valle, International Journal of Modern Physics D 20, 1745 (2011)

[38] R. Ruffini, M.G. Bernardini, C.L. Bianco, L. Caito, P. Chardonnet, M.G. Dainotti, R. Fraschetti, R. Guida, G. Vereshchagin, S. Xue, The Role of GRB 031203 in Clarifying the Astrophysical GRB Scenario, in ESA Special Publication (2007), Vol. 622 of ESA Special Publication, p. 561, 0705.2456

[39] J.A. Rueda, R. Ruffini, ApJ 758, L7 (2012)

[40] C.L. Fryer, J.A. Rueda, R. Ruffini, ApJ Letters 793, L36 (2014), 1409. 1473

[41] L. Becerra, F. Cipolletta, C.L. Fryer, J.A. Rueda, R. Ruffini, ApJ 812, 100 (2015), 1505.07580

[42] R. Ruffini, J.A. Rueda, M. Muccino, Y. Aimuratov, L.M. Becerra, C.L. Bianco, M. Kovacevic, R. Moradi, F.G. Oliveira, G.B. Pisani et al., ApJ 832, 136 (2016), 1602.02732

[43] J. Heise, X-Ray Flashes and X-Ray Counterparts of Gamm-Ray Bursts, in Gamma-Ray Burst and Afterglow Astronomy 2001: A Workshop Celebrating the First Year of the HETE Mission, edited by G.R. Ricker, R.K. Vanderspek (2003), Vol. 662 of American Institute of Physics Conference Series, pp. 229236, astro-ph/0111246

[44] L. Amati, F. Frontera, J.J.M. in't Zand, M. Capalbi, R. Landi, P. Soffitta, L. Vetere, L.A. Antonelli, E. Costa, S. Del Sordo et al., A\&A 426, 415 (2004), astro-ph/0407166

[45] A.M. Soderberg, S.R. Kulkarni, E. Nakar, E. Berger, P.B. Cameron, D.B. Fox, D. Frail, A. Gal-Yam, R. Sari, S.B. Cenko et al., Nature 442, 1014 (2006), astro-ph/0604389

[46] G.B. Pisani, L. Izzo, R. Ruffini, C.L. Bianco, M. Muccino, A.V. Penacchioni, J.A. Rueda, Y. Wang, A\&A 552, L5 (2013), 1304. 1764

[47] R. Ruffini, M. Muccino, C.L. Bianco, M. Enderli, L. Izzo, M. Kovacevic, A.V. Penacchioni, G.B. Pisani, J.A. Rueda, Y. Wang, A\&A 565, L10 (2014), 1404.3946

[48] C.L. Fryer, F.G. Oliveira, J.A. Rueda, R. Ruffini, Physical Review Letters 115, 231102 (2015), 1505.02809

[49] L. Becerra, C.L. Bianco, C.L. Fryer, J.A. Rueda, R. Ruffini, ApJ 833, 107 (2016), 1606.02523

[50] R. Giacconi, R. Ruffini, Physics and astrophysics of neutron stars and black holes (1978)

[51] I.F. Mirabel, L.F. Rodríguez, Nature 392, 673 (1998)

[52] L. Caito, M.G. Bernardini, C.L. Bianco, M.G. Dainotti, R. Guida, R. Ruffini, A\&A 498, 501 (2009), Q810. 4855

[53] L. Caito, L. Amati, M.G. Bernardini, C.L. Bianco, G. de Barros, L. Izzo, B. Patricelli, R. Ruffini, A\&A 521, A80+ (2010), 1006. 4842

[54] M. Della Valle, G. Chincarini, N. Panagia, G. Tagliaferri, D. Malesani, V. Testa, D. Fugazza, S. Campana, S. Covino, V. Mangano et al., Nature 444, 1050 (2006), arXiv:astro-ph/0608322

[55] Z.P. Jin, X. Li, Z. Cano, S. Covino, Y.Z. Fan, D.M. Wei, ApJ Letters 811, L22 (2015), 1507.07206

[56] M. Cadelano, C. Pallanca, F.R. Ferraro, M. Salaris, E. Dalessandro, B. Lanzoni, P.C.C. Freire, ApJ 812, 63 (2015), 1509.01397

[57] T.M. Tauris, E.P.J. van den Heuvel, G.J. Savonije, ApJ Letters 530, L93 (2000), astro-ph/0001013

[58] P. Lazarus, T.M. Tauris, B. Knispel, P.C.C. Freire, J.S. Deneva, V.M. Kaspi, B. Allen, S. Bogdanov, S. Chatterjee, I.H. Stairs et al., MNRAS 437, 1485 (2014), 1310. 5857

[59] R. Ruffini, J.D. Salmonson, J.R. Wilson, S.S. Xue, A\&A 350, 334 (1999), arXiv: astro-ph/9907030

[60] R. Ruffini, J.D. Salmonson, J.R. Wilson, S. Xue, A\&A 359, 855 (2000), arXiv: astro-ph/0004257

[61] A.G. Aksenov, R. Ruffini, G.V. Vereshchagin, Physical Review Letters 99, 125003 (2007), 0707. 3250

[62] A.G. Aksenov, R. Ruffini, G.V. Vereshchagin, Physical Review D 79, 043008 (2009), 0901. 4837

[63] M. Muccino, R. Ruffini, C.L. Bianco, L. Izzo, A.V. Penacchioni, ApJ 763, 125 (2013), 1205.6600

[64] R. Ruffini, M. Muccino, Y. Aimuratov, C.L. Bianco, C. Cherubini, M. Enderli, M. Kovacevic, R. Moradi, A.V. Penacchioni, G.B. Pisani et al., ApJ 831, 178 (2016), 1607.02400

[65] Y. Aimuratov, R. Ruffini, M. Muccino, C.L. Bianco, A.V. Penacchioni, G.B. Pisani, D. Primorac, J.A. Rueda, Y. Wang, ApJ 844, 83 (2017), 1704. 08179 
[66] R. Ruffini, C.L. Bianco, P. Chardonnet, F. Fraschetti, S. Xue, ApJ Letters 581, L19 (2002), arXiv: astro-ph/0210648

[67] R. Ruffini, C.L. Bianco, S.S. Xue, P. Chardonnet, F. Fraschetti, V. Gurzadyan, International Journal of Modern Physics D 13, 843 (2004), arXiv: astro-ph/0405284

[68] R. Ruffini, M.G. Bernardini, C.L. Bianco, P. Chardonnet, F. Fraschetti, V. Gurzadyan, L. Vitagliano, S.S. Xue, The Blackholic energy: long and short Gamma-Ray Bursts (New perspectives in physics and astrophysics from the theoretical understanding of Gamma-Ray Bursts, II), in XIth Brazilian School of Cosmology and Gravitation, edited by M. Novello \& S. E. Perez Bergliaffa (2005), Vol. 782 of American Institute of Physics Conference Series, p. 42, arXiv: astro-ph/0503476

[69] C.L. Bianco, R. Ruffini, ApJ Letters 633, L13 (2005), arXiv: astro-ph/0509621

[70] C.L. Bianco, R. Ruffini, ApJ Letters 620, L23 (2005), arXiv: astro-ph/0501390

[71] R. Narayan, B. Paczynski, T. Piran, ApJ Letters 395, L83 (1992), arXiv: astro-ph/9204001

[72] J.D. Salmonson, J.R. Wilson, ApJ 578, 310 (2002), astro-ph/0203349

[73] Y.B. Zel'dovich, L.N. Ivanova, D.K. Nadezhin, Soviet Astronomy 16, 209 (1972)

[74] R. Ruffini, J. Wilson, Physical Review Letters 31, 1362 (1973)

[75] K. Maeda, K. Nomoto, ApJ 598, 1163 (2003), astro-ph/0304172

[76] T.J. Galama, P.M. Vreeswijk, J. van Paradijs et al., Nature 395, 670 (1998)

[77] S. Campana, V. Mangano, A.J. Blustin, P. Brown, D.N. Burrows, G. Chincarini, J.R. Cummings, G. Cusumano, M. Della Valle, D. Malesani et al., Nature 442, 1008 (2006), arXiv: astro-ph/0603279

[78] R. Ruffini, Black Holes, Supernovae and Gamma Ray Bursts, in Thirteenth Marcel Grossmann Meeting: On Recent Developments in Theoretical and Experimental General Relativity, Astrophysics and Relativistic Field Theories, edited by K. Rosquist (2015), pp. 242-314

[79] R. Ruffini, Y. Wang, Y. Aimuratov, L. Becerra, C.L. Bianco, M. Karlica, M. Kovacevic, L. Li, J.D. Melon Fuksman, R. Moradi et al., ArXiv e-prints (2017), 1704.03821

[80] R. Ruffini, C.L. Bianco, M. Enderli, M. Muccino, A.V. Penacchioni, G.B. Pisani, J.A. Rueda, N. Sahakyan, Y. Wang, L. Izzo, GRB Coordinates Net- work 14526, 1 (2013)

[81] A. de Ugarte Postigo, D. Xu, G. Leloudas, T. Kruehler, D. Malesani, J. Gorosabel, C.C. Thoene, R. Sanchez-Ramirez, S. Schulze, J.P. Fynbo et al., GRB Coordinates Network 14646, 1 (2013)

[82] A.J. Levan, A.S. Fruchter, J. Graham, N.R. Tanvir, J. Hjorth, J. Fynbo, D. Perley, S.B. Cenko, E. Pian, Z. Cano et al., GRB Coordinates Network 14686, 1 (2013)

[83] A.M. Watson, N. Butler, A. Kutyrev, W.H. Lee, M.G. Richer, C. Klein, O. Fox, J.X. Prochaska, J. Bloom, A. Cucchiara et al., GRB Coordinates Network 14666, 1 (2013)

[84] D. Xu, A. de Ugarte Postigo, T. Kruehler, D. Malesani, G. Leloudas, J.P.U. Fynbo, J. Hjorth, S. Schulze, P. Jakobsson, Z. Cano et al., GRB Coordinates Network 14597, 1 (2013)

[85] L. Nava, G. Vianello, N. Omodei, G. Ghisellini, G. Ghirlanda, A. Celotti, F. Longo, R. Desiante, R. Barniol Duran, MNRAS 443, 3578 (2014), 1406.6693

[86] M. Ackermann, M. Ajello, K. Asano, M. Axelsson, L. Baldini, J. Ballet, G. Barbiellini, D. Bastieri, K. Bechtol, R. Bellazzini et al., ApJ Supplement 209, 11 (2013), 1303. 2908

[87] F. Cipolletta, C. Cherubini, S. Filippi, J.A. Rueda, R. Ruffini, Physical Review D 92, 023007 (2015), 1506.05926

[88] E. Troja, S. Rosswog, N. Gehrels, ApJ 723, 1711 (2010), 1009.1385

[89] R. Belvedere, J.A. Rueda, R. Ruffini, Journal of Korean Physical Society 65, 897 (2014)

[90] Y. Lithwick, R. Sari, ApJ 555, 540 (2001)

[91] L.D. Landau, E.M. Lifshitz, The classical theory of fields (Course of theoretical physics - Pergamon International Library of Science, Technology, Engineering and Social Studies, Oxford: Pergamon Press, 4th rev.engl.ed., 1975)

[92] K. Boshkayev, J.A. Rueda, R. Ruffini, I. Siutsou, ApJ 762, 117 (2013), 1204.2070

[93] R. Ruffini, G. Vereshchagin, S. Xue, Physics Reports 487, 1 (2010), 0910.0974

[94] F. Özel, P. Freire, Annual Review of A\&A 54, 401 (2016), 1603.02698

[95] F.G. Oliveira, J.A. Rueda, R. Ruffini, ApJ 787, 150 (2014)

[96] R. Ruffini, J. Rodriguez, M. Muccino, J.A. Rueda, Y. Aimuratov, U. Barres de Almeida, L. Becerra, C.L. Bianco, C. Cherubini, S. Filippi et al., ArXiv e-prints (2016), 1602.03545 